\title{
Cerebral Neurovascular Coupling Impairment in Central Serous Chorioretinopathy
}

\author{
Susana Penas ${ }^{a, b}$ Pedro Castro ${ }^{b, c}$ Gilberto Pereira ${ }^{c}$ Ana Marta Oliveira ${ }^{d}$ \\ Angela M. Carneiro ${ }^{a, b}$ Amândio Rocha-Sousa ${ }^{a, b}$ Elsa Azevedo ${ }^{b, c}$ \\ Fernando M. Falcão-Reis ${ }^{a, b}$ \\ aOphthalmology Department, Centro Hospitalar Universitário de São João, E.P.E., Porto, Portugal; \\ ${ }^{b}$ Faculty of Medicine of University of Porto, Porto, Portugal; ' $N$ Neurology Department, Centro Hospitalar \\ Universitário de São João, E.P.E., Porto, Portugal; 'Investigation Unit, Centro Hospitalar Universitário \\ de São João, E.P.E., Porto, Portugal
}

\section{Keywords}

Central serous chorioretinopathy $\cdot$ CSC $\cdot$ Neurovascular coupling $\cdot$ Cerebrovascular regulation

\begin{abstract}
Background: Central serous chorioretinopathy (CSCR) is a chorioretinal disorder resulting from choroidal hyperpermeability. Its comorbidities as hypertension, coronary disease, and psychological stress, suggest that it might reflect a more generalized vascular dysfunction. Objectives: The aim of the study was to assess the cerebrovascular regulation integrity, using cerebral autoregulation (CA), carbon dioxide vasoreactivity (VR), and neurovascular coupling (NVC) in CSCR. Methods: This observational pilot study included $20 \mathrm{CSCR}$ patients and 14 age- and sex-matched controls. A State-Trait Anxiety Inventory (STAI) inquiry was full-filled. Continuous measurement of cerebral blood flow velocity (CBFV), arterial blood pressure, heart rate, and end-tidal carbon dioxide was performed. VR was assessed during hypercapnia (inhaling carbogen gas) and hypnocapnia (hyperventilation). For NVC, the $\mathrm{CBFV}$ relative increase during mental activation using
\end{abstract}

the N-Back Task was calculated. Results: No significant differences in systemic hemodynamic parameters, CA or VR, were found between both groups. During the NVC performance, the average CBFV rise during mental stress was significantly lower in CSCR $(p=0.011)$. A significant negative correlation was found between STAI scores and NVC. Conclusions: CSCR patients presented a significantly impaired cerebral NVC compared to controls, supporting the theory of a potential systemic vascular dysfunction. Stress could be related to this NVC impairment. @2020 The Author(s).

Published by S. Karger AG, Basel

\section{Introduction}

Central serous chorioretinopathy (CSCR) is a chorioretinal disorder characterized by one or multiple serous detachments of the neurosensory retina. It is more frequent among middle-aged male individuals and has been described in patients with specific psychological and personality profiles, related to stress, anxiety, and Type-A behavior [1].

C 2020 The Author(s).

Published by S. Karger AG, Basel

This is an Open Access article licensed under the Creative Commons Attribution-NonCommercial-4.0 International License (CC BY-NC) (http://www.karger.com/Services/OpenAccessLicense), applicable to the online version of the article only. Usage and distribution for commercial purposes requires written permission.
Correspondence to:

Susana Penas, susanaapenas@gmail.com 
Its pathophysiology remains poorly understood, but recent multimodal imaging highlighted the role of a choroidal vascular hyperpermeability. Recently the "pachychoroid" terminology has been proposed for a spectrum of diseases, including CSCR, characterized by the presence of a thick choroid with abnormal dilated choroidal vessels in Haller's layers (pachyvessels) and compressed choriocapillaris and Sattler's layers [2-4].

The relation between CSCR and steroids has been widely recognized and is probably one of the most intriguing aspects of the disease [5-7]. A significant sympathetic-parasympathetic imbalance has also been described in CSCR patients, presenting an overactive sympathetic and an under-reactive parasympathetic profile, assessed by blood pressure and heart rate (HR) variability $[8,9]$. Furthermore, an increased incidence of cardiovascular events has been found in these patients, presenting a higher rate of coronary heart disease [10] or ischemic stroke [11].

However, the precise mechanisms relating CSCR to anxiety-sensitive personalities, steroid biochemistry, dysautonomia, and cardiovascular risk factors are far from being elucidated, and there are probably many missing factors in this complex equation. It seems that the pathogenesis of CSCR might extend beyond a local choroidal vascular disorder and is possibly the reflection of a systemic vascular dysfunction [5]. This study aimed the assessment of the systemic hemodynamic parameters and cerebrovascular regulation in CSCR patients, comparing to controls.

\section{Material and Methods}

\section{Study Participants}

A sample of 20 consecutive patients from our retinal department with a diagnosis of CSCR was selected. Both patients with active (acute and chronic) and inactive CSCR were included. An active disease status was described for patients with a history of a serous retinal detachment on optical coherence tomography and leakage on fluorescein angiography within 3 or less months from this assessment. Patients presenting a resolution of a serous retinal detachment for more than 3 months from baseline assessment were classified as inactive CSCR.

A sample of 14 control healthy volunteers, with similar age and sex ratios, was selected. Exclusion criteria for both groups included systemic relevant cardiovascular diseases as hypertension, ischemic coronary heart disease or stroke, diabetes, neuropsychiatric disorder, use of corticosteroids or vasoactive medications, or other ocular diseases besides CSCR. The study guidelines were explained to all the participants and they were given written information explaining the study procedures. A written consent was obtained before their inclusion. All the participants initially filled in a Portuguese-translated State-Trait Anxiety Inventory (STAI) or STAI-Y1 form to measure distress and diagnose trait and state anxiety [12-14]. The STAI S (state) form was presented first, followed by the STAI T (trait) form. An STAI Anxiety State score $>47$ and an STAI Anxiety Trait score $>42$ were considered pathological, according to the cut-offs established by the author, describing a high level of anxiety. The study design and the collection of data were approved by the Ethics Committee of our hospital. All procedures were conducted in accordance with the Declaration of Helsinki.

\section{Monitoring Protocol}

The monitoring protocol used was previously described by one of the authors [15]. Patients were evaluated in a dim-lighted room, with a temperature of around $20^{\circ} \mathrm{C}$, in a supine position, with the bed head at $0^{\circ}$. Cerebral blood flow velocity (CBFV) was recorded bilaterally from the M1 segment of the middle cerebral artery at a depth of $50-55 \mathrm{~mm}$, using $2-\mathrm{MHz}$ monitoring probes properly secured by an individually fitted headband (Doppler-Box X; DWL, Singen, Germany). Continuous arterial blood pressure (ABP) was recorded using a finger cuff Finometer MIDI device (FMS, Amsterdam, The Netherlands) on the nondominant side. HR was assessed from lead II of a standard 3-lead electrocardiogram. Endtidal carbon dioxide $\left(\mathrm{EtCO}_{2}\right)$ was recorded using a nasal cannula with a capnograph (RespSense; Nonin, Amsterdam, The Netherlands). All data were synchronized and digitized at $400 \mathrm{~Hz}$ with PowerLab (AD Instruments, Oxford, UK) and stored for offline analysis. After a 20 -min rest, a 10 -min period of resting data was stored for cerebral autoregulation (CA) calculations. Afterward, carbon dioxide vasoreactivity (VR) and neurovascular coupling (NVC) protocols were performed.

\section{CA Calculations}

To assess CA, the mean values of ABP and CBFV were calculated for each heartbeat. Dynamic CA was assessed by transfer function analysis (TFA), which was done by calculating the coherence, gain, and phase parameters from beat-to-beat spontaneous oscillations in CBFV and ABP. TFA parameter settings were in compliance with standard recommendations [16]: Lower coherence (correlation coefficient), lower gain (damping of arterial BP oscillations), and higher phase (speed of the autoregulatory response) between oscillations of ABP and mean CBFV indicate more effective CA. Since vasomotor adaptation is slow and requires at least 6-10 s, CA is most likely to operate at low frequencies $[17,18]$. Values were therefore reported in the 2 bands with lower frequencies: very low frequencies (VLF: 0.02-0.07 Hz) and low frequencies (LF: 0.07-0.20 Hz).

\section{VR Protocol}

On a second stage of our study, and after resting, all subjects breathed carbogen gas (Carbox $5 \mathrm{CO}_{2} 5 \%$ and $\mathrm{O}_{2} 95 \%$ ), delivered through a partially expanded reservoir bag at atmospheric pressure, for $2 \mathrm{~min}$. After stabilization of hemodynamic parameters back to baseline, they hyperventilated to an $\mathrm{EtCO}_{2} \sim 20 \mathrm{~mm} \mathrm{Hg}$ for another $2 \mathrm{~min}$. VR was calculated as the slope of the relationship between $\mathrm{EtCO}_{2}$ plotted against relative MFV at the last $30 \mathrm{~s}$ of hypocapnia or hypercapnia and expressed as change \% of the MFV/ $\mathrm{mm} \mathrm{Hg}$ of $\mathrm{CO}_{2}$. VR was also calculated separately for hypercapnia and hypocapnia. 
Table 1. Clinical and demographic characteristics of the participants

\begin{tabular}{llll}
\hline & \multicolumn{2}{l}{ Clinical and demographic characteristics } & \\
\cline { 2 - 4 } & CSCR & controls & $p$ value \\
\hline$N$ & 20 & 14 & - \\
Age, years & $47.0 \pm 9.6$ & $45.9 \pm 9.2$ & $0.756^{\dagger}$ \\
Sex male/female, $n(\%)$ & $16(80) / 4(20.0)$ & $10(71.4) / 4(28.6)$ & $0.562^{\ddagger}$ \\
Active CSCR, $n(\%)$ & $10(50)$ & - & - \\
Inactive CSCR, $n(\%)$ & $10(50)$ & - & \\
STAI Anxiety State score & $38.5( \pm 8.4)$ & $25.0( \pm 4.8)$ & $0.002^{\S *}$ \\
STAI Anxiety Trait score & $44.5( \pm 4.9)$ & $32.0( \pm 4.6)$ & $0.002^{\S *}$ \\
\hline
\end{tabular}

Values are presented as mean \pm standard deviation (SD). CSCR, central serous chorioretinopathy; STAI, State-Trait Anxiety Inventory. ${ }^{*} p<0.05 . p$ value for ${ }^{\dagger} T$ test, ${ }^{\ddagger} X^{2}$ Test, $\S$ Mann-Whitney.

\section{NVC Protocol}

The N-Back Task was performed and analyzed as by Sorond et al. [19]. While in supine position, a sequence of single letters was displayed onto the ceiling. Subjects were first instructed to press the mouse button each time a letter was repeated (1-Back) and then each time a letter was repeated every other letter (2-Back). A control task was performed before each task - "Identify the letter X"; NVC was calculated as the ratio of the relative MFV increase during the N-Back (MFVNB) compared with the control task of "Identify the letter X" (MFVIDX) using the following formula: [(MFVNB - MFVIDX)/(MFVIDX)] × 10 [19]. Only the 2-Back Task results were reported, since they induce a higher CBFV rise and are potentially more representative for NVC assessment [20].

\section{Statistics}

For statistical analysis, SPSS software version 24.0 (SPSS Inc, Chicago, IL, USA) was used. Shapiro-Wilk test was used to assess the normality of the sample. Comparisons between groups were performed using $T$ test, Mann-Whitney, and $\chi^{2}$ test, as appropriate. The correlation analysis was performed using the Spearman's test.

\section{Results}

Table 1 summarizes the clinical and demographic data of the participants. Twenty patients with CSCR and 14 healthy controls were included. The mean age of the CSCR group was $47.0+9.6$ years and in the control group was $45.9+9.2$ years. The majority of the participants were male (76.5\%) (26 male - 16 CSCR patients and 10 controls). Eight (23.5\%) female patients were included (4 CSCR patients and 4 controls).

A significant difference was found for mean STAI scores, both for Anxiety State and Anxiety Trait, between CSCR patients and controls $(p=0.002)$ (Table 1). An STAI score $>47$ for the Anxiety State was found in 25\% ( $n$ $=5$ ) of CSCR patients and in none of the controls. An
Table 2. Baseline hemodynamics of the participants

\begin{tabular}{llll}
\hline & \multicolumn{3}{l}{ Baseline hemodynamics } \\
\cline { 2 - 4 } & CSCR patients & controls & $p$ value \\
\hline Mean ABP, mm Hg & $88.2 \pm 14.7$ & $79.3 \pm 9.90$ & $0.061^{\dagger}$ \\
HR, bpm & $69.5 \pm 14.6$ & $63.8 \pm 12.3$ & $0.220^{\ddagger}$ \\
ET CO $2, \mathrm{~mm} \mathrm{Hg}^{-1}$ & $37.6 \pm 3.90$ & $39.4 \pm 2.34$ & $0.165^{\dagger}$ \\
CBFV, $\mathrm{cm} \mathrm{sec}^{-1}$ & $56.4 \pm 7.01$ & $58.5 \pm 8.02$ & $0.377^{\ddagger}$ \\
\hline
\end{tabular}

Values are presented as mean \pm standard deviation (SD). CSCR, central serous chorioretinopathy; $B P$, blood pressure; $H R$, heart rate; $\mathrm{EtCO}_{2}$, end-tidal $\mathrm{CO}_{2}$. $p$ value for ${ }^{\dagger} T$ test, ${ }^{\ddagger}$ Mann-Whitney.

STAI score $>42$ for the Anxiety Trait was registered in $55 \%(n=11)$ of the patients and only in $7 \%(n=1)$ of the controls. A significant negative correlation was found between STAI scores for both groups $(r=-0.730 p<0.01)$.

In the resting condition (Table 2), although slightly higher in CSCR patients, no significant differences were recorded for mean $\mathrm{ABP}$ or $\mathrm{HR}(p=0.061$ and $p=0.220$, respectively). In a subgroup analysis for the activity of disease, active CSCR patients presented a higher mean ABP than inactive patients $(p<0.05)$, but no other relevant differences were found. The $\mathrm{EtCO}_{2}$ measurement was similar between both patients and controls $(p=$ $0.165)$, as well as the mean CBFV $(p=0.377)$ (Table 2). Regarding CA (shown in Fig. 1; Table 3), both groups presented a similar performance in both VLF and LF average coherence, phase, and gain, meaning that the buffering of beat-to-beat spontaneous oscillation of mean ABP was preserved in CSCR patients when compared to controls.

Moreover, the VR response (shown in Fig. 2; Table 3), both in hypercapnia (inhaling carbogen) and hypnocapnia (hyperventilation) conditions, proved to be no differ- 


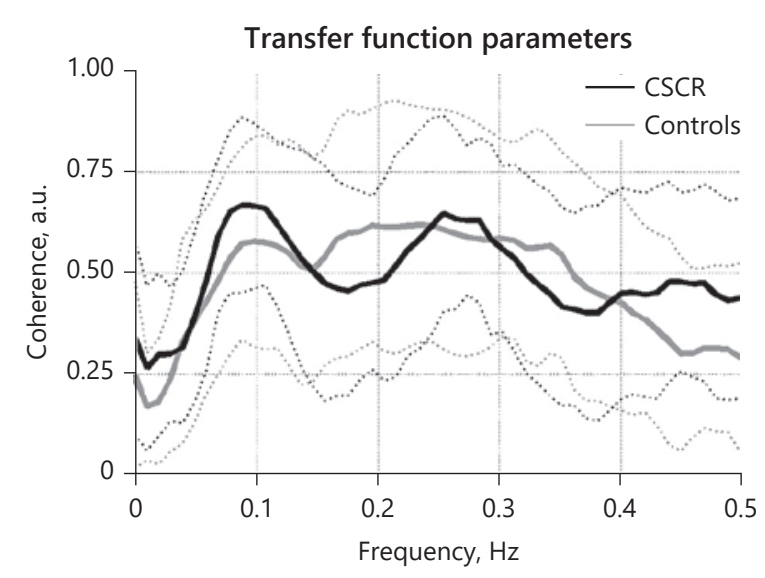

ent in CSCR versus controls $(p=0.914$ and $p=0.822$, respectively). Mean global VR, although higher in controls, was also similar in both groups $(p=0.822)$.

However, a significant difference between CSCR patients and controls was detected in the cerebrovascular hemodynamics assessment during NVC performance (shown in Fig. 3; Table 3). The mean CBFV gain during the 2-Back Task was significantly lower in CSCR patients $(p=0.011)$. Interestingly, this test had no different repercussion on systemic ABP, $\mathrm{HR}$, and $\mathrm{EtCO}_{2}$ across both groups. Similar NVC performances were found in both active and inactive CSCR patients $(p=0.619)$. A negative correlation was found between both the STAI scores and the NVC, more significant for the Anxiety State than the Anxiety Trait score $(r=-0.602, p=0.002$ and $-0.442, p=$

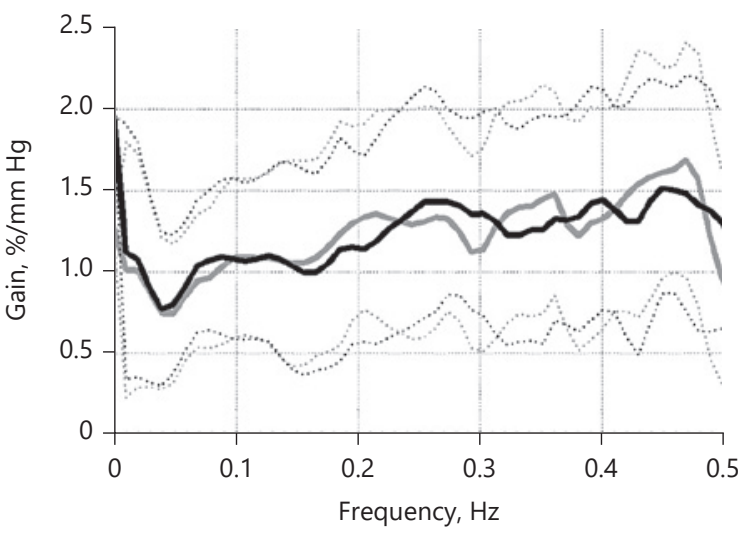
0.035) (shown in Fig. 4).

\section{Discussion}

In our study, we found that CSCR patients presented a significantly impaired cerebrovascular regulation that affects primarily the NVC. So far to our knowledge, this is the first report on the cerebrovascular regulation performance in CSCR disease.

A competent cerebrovascular regulation is mainly conducted by three major physiological mechanisms: CA, VR, and NVC. We found baseline hemodynamic measurements to be similar in CSCR patients and controls, as well as the cerebrovascular reaction to different levels of $\mathrm{CO}_{2}$. CSCR patients' cerebral blood vessels presented an equally efficient capacity to adapt to $\mathrm{ABP}$ fluctuations as controls (CA).

When submitted to a vasomotor stimulation during $\mathrm{VR}$, using a vasoactive substance like $\mathrm{CO}_{2}$, the increase in cerebral flow (or flow velocity) resulting from hypercapnia conditions and its decrease conditioned by hypocapnia was not significantly different in CSCR patients when

Fig. 1. Dynamic CA performance. Dynamic CA assessed by TFA from beat-to-beat spontaneous oscillations in CBFV (output) and $\mathrm{ABP}$ (input). Coherence, gain, and phase are represented along all spectrum of frequencies of oscillations. CA acts as a high-pass filter below frequencies around $0.2-0.3 \mathrm{~Hz}$, at which gain is reduced (amplitude of transmission between ABP to CBFV) and phase increases (higher desynchronization between $\mathrm{ABP}$ and CBFV oscillations). CA in CSCR patients (thick black line) and controls (thick gray line) was not significantly different. Black and gray dotted lines represent mean \pm SE in CSCR and controls, respectively. SE, standard error.

Cerebral Neurovascular Coupling in CSCR

Fig. 2. Time course of normalized $\mathrm{CBFV}$ and $\mathrm{EtCO}_{2}$ during hyper and hypnocapnia. Time course of normalized mean $\operatorname{CBFV}(\mathbf{a}, \mathbf{c})$ and mean $\mathrm{EtCO}_{2}(\mathbf{b}, \mathbf{d})$ during the hypercapnic challenge (left) and hyperventilation-induced hypocapnia (right) of CSCR patients (thick black line) and controls (thick gray line); black and gray dotted lines represent mean \pm SE, respectively. The beginning and ending of each task are marked with vertical black lines. No significant differences were found between both groups. CBFV, cerebral blood flow velocity; $\mathrm{EtCO}_{2}$, end-tidal $\mathrm{CO}_{2}$; CSCR, central serous chorioretinopathy; $\mathrm{SE}$, standard error.

(For figure see next page.) 
Table 3. Cerebrovascular regulation performance of the participants

\begin{tabular}{|c|c|c|c|}
\hline Cerebrovascular regulation & CSCR patients & controls & $p$ value \\
\hline \multicolumn{4}{|l|}{$C A$} \\
\hline Coherence VLF, a.u & $0.41 \pm 0.19$ & $0.35 \pm 0.16$ & $0.302^{\dagger}$ \\
\hline Coherence LF, a.u & $0.59 \pm 0.24$ & $0.55 \pm 0.20$ & $0.635^{\dagger}$ \\
\hline Phase VLF, radians & $0.95 \pm 0.38$ & $0.98 \pm 0.48$ & $0.248^{\dagger}$ \\
\hline Phase $L F$, radians & $0.78 \pm 0.19$ & $0.76 \pm 0.31$ & $0.363^{\dagger}$ \\
\hline Gain VLF, \% $\mathrm{mm} \mathrm{Hg}^{-1}$ & $0.78 \pm 0.29$ & $0.84 \pm 0.39$ & $0.320^{\dagger}$ \\
\hline Gain LF, \% mm Hg & $1.31 \pm 0.38$ & $1.07 \pm 0.52$ & $0.277^{\dagger}$ \\
\hline \multicolumn{4}{|l|}{ VR } \\
\hline VR in hypercapnia, \% mm Hg & $3.85 \pm 1.50$ & $3.97 \pm 1.75$ & $0.914^{\ddagger}$ \\
\hline VR in hypnocapnia, $\% \mathrm{~mm} \mathrm{Hg}^{-1}$ & $3.28 \pm 2.67$ & $2.85 \pm 2.21$ & $0.822^{\ddagger}$ \\
\hline Global VR, \% $\mathrm{mm} \mathrm{Hg}^{-1}$ & $1.35 \pm 0.61$ & $1.67 \pm 0.46$ & $0.139^{\dagger}$ \\
\hline \multicolumn{4}{|l|}{ NVC } \\
\hline Relative CBFV change in 2-back task, \% & $3.77 \pm 3.36$ & $7.77 \pm 4.05$ & $0.011^{\dagger *}$ \\
\hline
\end{tabular}

Values are presented as mean \pm standard deviation (SD). CSCR, central serous chorioretinopathy; a.u., arbitrary units; LF, low frequencies $(0.07-0.20 \mathrm{~Hz})$; VLF, very low frequencies (0.02-0.07 Hz); 2-Back gain - Increase of mean CBFV during 2-back task (\%). ${ }^{*} p<0.05$. $p$ value for ${ }^{\dagger} T$ test, ${ }^{\ddagger}$ Mann-Whitney.
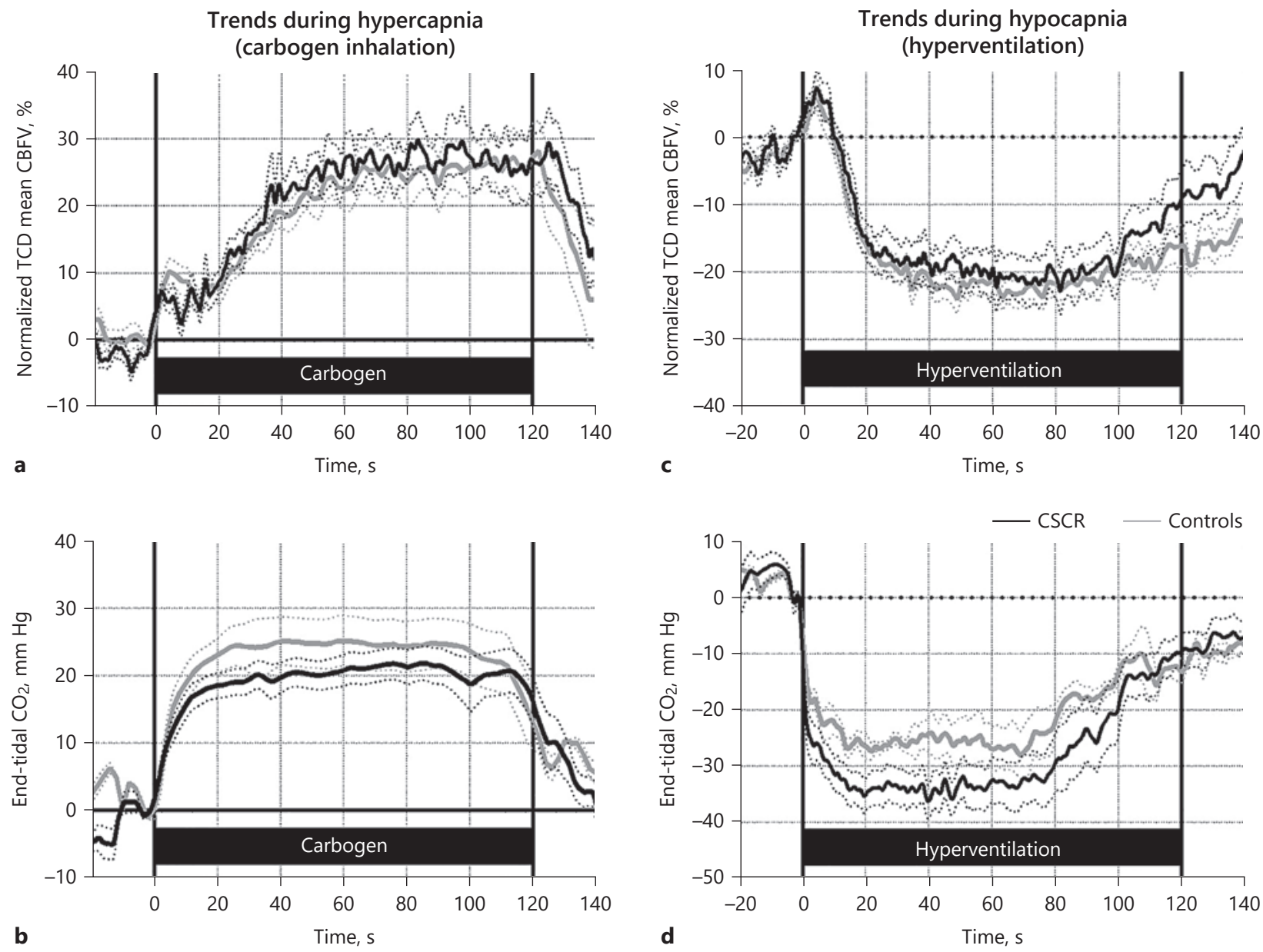


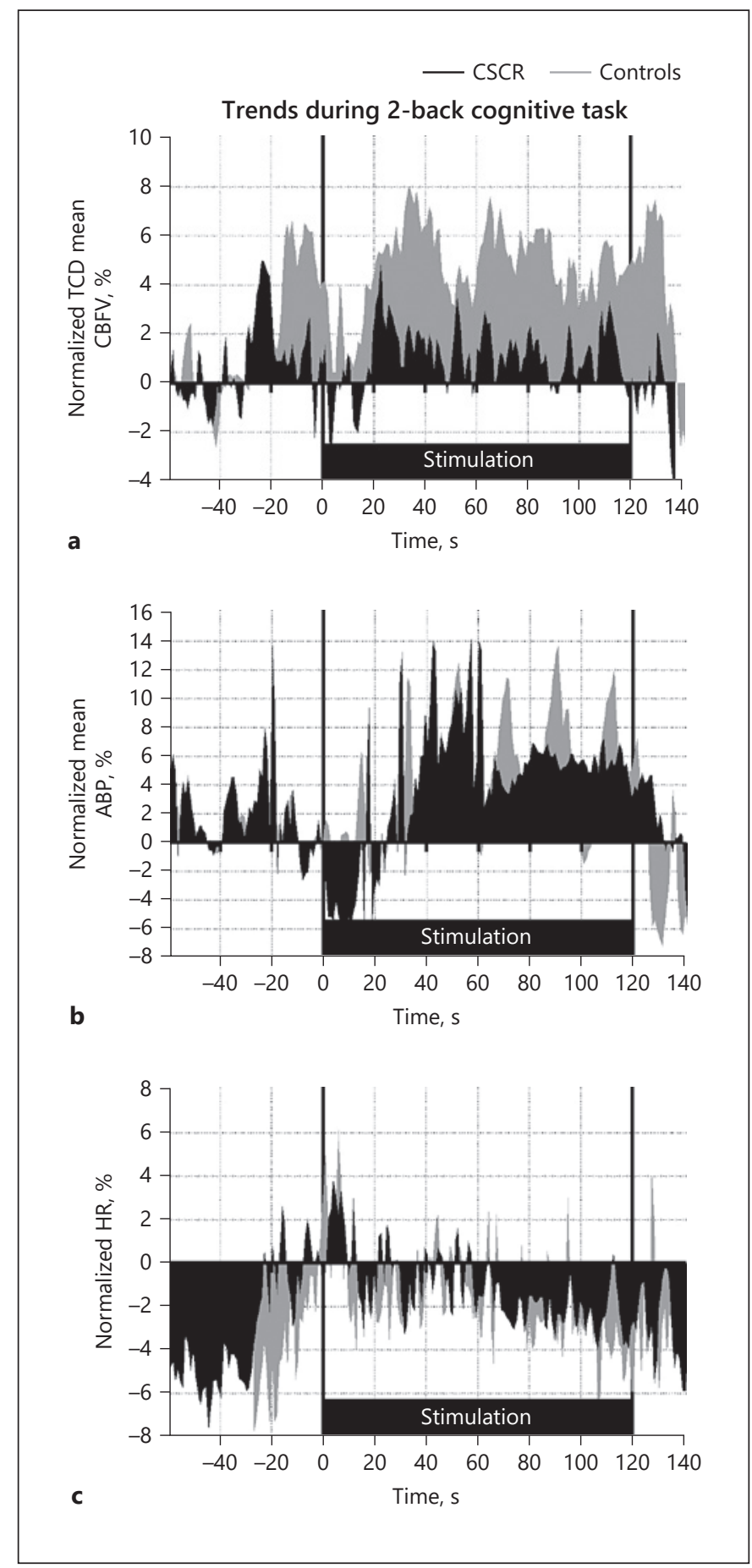

Fig. 3. Time course of normalized CBFV, ABP, and HR during the 2-Back Task. Group-averaged time course of normalized mean CBFV (a), mean ABP (b), HR (c) during the 2-Back Task, to assess NVC. Gray-shaded regions represent mean \pm SE. The beginning and ending of the task are marked with vertical black lines. CSCR patients presented a blunted response of the mean CBFV during mental activation when compared to controls. ABP, arterial blood pressure; $\mathrm{CBFV}$, cerebral blood flow velocity; $\mathrm{HR}$, heart rate; SE, standard error.

Cerebral Neurovascular Coupling in CSCR compared to controls. However, while testing cerebral functional hyperemia under a mental stress task as the 2-Back Task, the average CBFV increase proved to be significantly inferior in CSCR patients. This impairment was independent of the activity state of the disease, suggesting it could be inherent to this pathological condition.

Actually, Tomasso et al. [21] have recently described an impaired retinal NVC in CSCR, presenting a decreased dynamic vasodilation in response to light stimulation. They speculated that the decreased retinal vein dilation response to flicker light stimulation could possibly be due to an increased sympathetic tone and potentially lead to venous stasis and choroidal thickening. This retinal NVC impairment in CSCR corroborates our findings of a cerebral NVC impairment in these patients, since they share the same neurochemical mechanisms.

The concept of neurovascular unit represents the intricate relationship between the brain (or its neural activity) and its vessels. In the "resting" brain, cerebrovascular flow varies in proportion to the energy consumption of each brain region. If given a specific mental stress task, as the 2-Back Task used in our study, a functional hyperemia to the activated areas is expected. This happens due to the need of a sufficient delivery of oxygen and glucose, to provide for a low energy reserve tissue as the brain, as well as the need for a quick wash-out of potential toxic by-products resulting from cell activity (as lactate, $\mathrm{CO}_{2}$ ), and the need to regulate brain temperature [22]. Cerebrovascular delivery has been described to be regulated by a feedforward mechanism driven by neurovascular signaling pathways, resulting in the release of vasoactive byproducts of synaptic activity such as potassium $\left(\mathrm{K}^{+}\right)$, nitric oxide, and prostanoids [23].

We believe that higher levels of stress in CSCR patients could be related to their NVC impairment. The association of CSCR to stress and anxiety has been widely recognized [1] and in our study, the STAI score proved to be significantly higher for both Anxiety State and Trait in CSCR patients when compared to controls (Fig. 4). We also found a significant negative correlation among the STAI score and NVC, meaning that individuals with a higher level of anxiety presented a worse NVC performance, supporting our theory (Fig. 4). Curiously, this does not seem to be an effect of HR or ABP surge during mental activation, which was similar between groups (Fig. 3). It has been proved that stress may influence the neurovascular unit functioning in rats, acting through a multifaceted effect [24-26]. In fact, stress can act as a negative modulator of the remodeling process involved in glial and vascular compartment [24], as well as causing a 


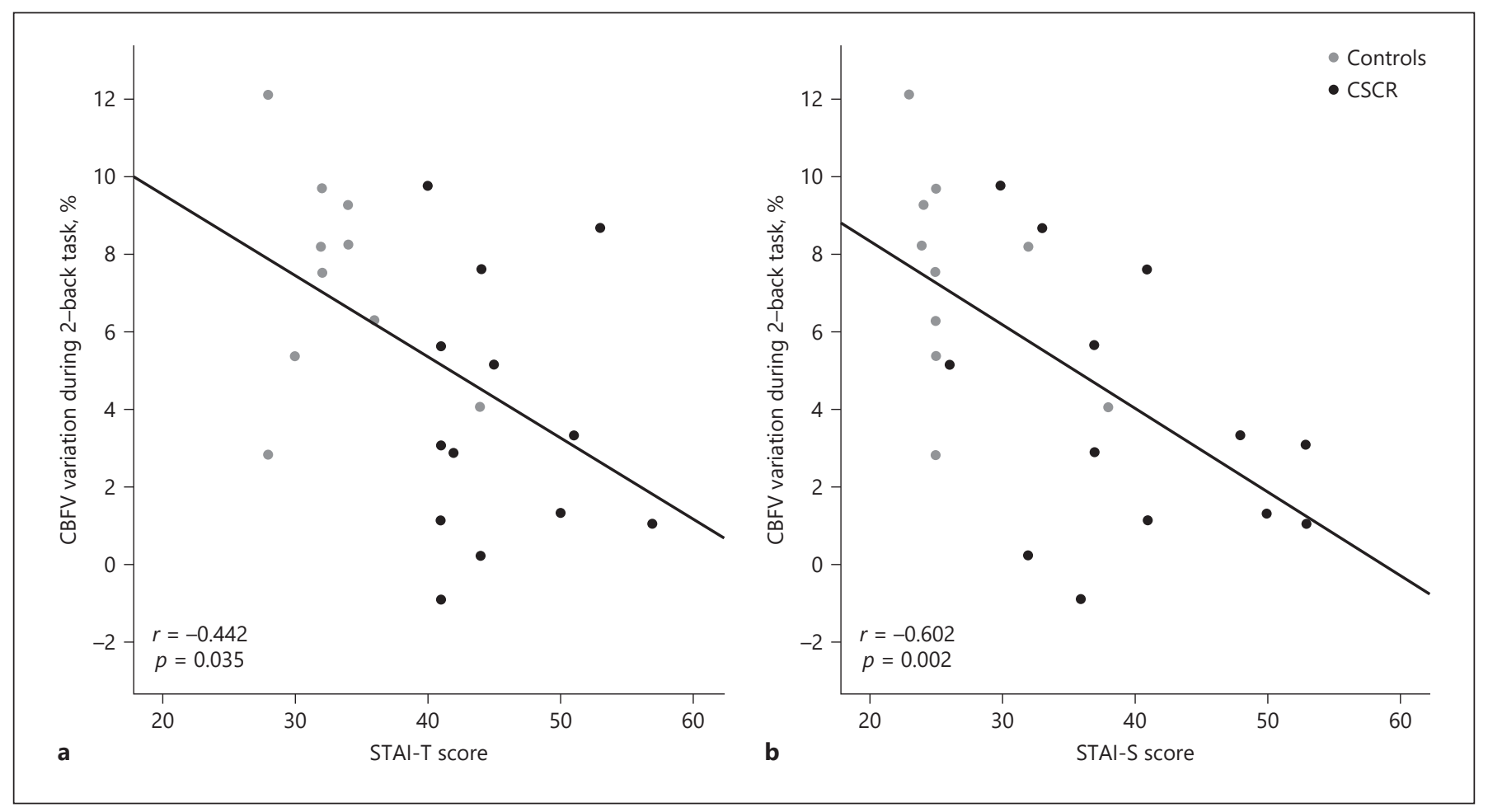

Fig. 4. Correlation between the STAI scores and neurovascular coupling. Graphs a, b representing the Spearman's rho negative correlation between the Anxiety Trait (STAI-T) and Anxiety State (STAI-S) score, respectively, and CBFV change during the 2-Back Task. Patients with CSCR (black dots) presented higher STAI scores than controls (gray dots). A more significant negative correlation was found for Anxiety State than Anxiety Trait.

glucocorticoid mediated disruption of $\mathrm{K}^{+}$-driven vasodilation, through impaired smooth muscle inwardly rectifying $\mathrm{K}^{+}$channel function [25]. This ultimately renders arterioles less sensitive to small increases in local $\mathrm{K}^{+}$released by astrocytes, causing impairment in $\mathrm{K}^{+}$-mediated vasodilation during $\mathrm{NVC}$, and, consequently, dampening the functional hyperemia. A NVC impairment has also recently been described in multiple pathologies with a recognized stress component, such as anxiety, depression, post-traumatic stress disorder, stroke, and Alzheimer's disease [20,22].

Considering the intricate relation of stress with autonomic nervous system activity, we could also speculate if the autonomic imbalance described in CSCR could have influenced our findings. An impaired NVC in the brain of patients with dysautonomia has been previously described by our investigation team [26]. Nevertheless, very little is known about the influence of autonomic imbalance in cerebrovascular regulation, rendering all the hypothetical attempts highly speculative.

There is nowadays a suspicion that CSCR may be associated with a more generalized vascular systemic dys- function, rather than just a localized choroidal malfunctioning $[5,27]$. An endothelial dysfunction could potentially be present in CSCR, since it has been described in several cardiovascular diseases such as coronary heart disease and stroke, also reported in CSCR patients $[5,10$, $11,27,28]$. More recently, the recognition of an association between the aldosterone/mineralocorticoid receptor pathway and CSCR has encouraged the use of mineralocorticoid receptors (MRs) antagonist in its treatment [5]. Increasing evidence suggests that blocking MR activation can have therapeutic value for endothelial dysfunction, atherosclerosis, hypertension, heart failure, and chronic kidney disease [5]. Inappropriate or overactivation of the MRs in ocular cells and other tissues, such as brain, heart, and systemic vessels, could relate CSCR pathology with its known comorbidities including hypertension, coronary disease and psychological stress. However, the VICI trial, a large randomized placebo controlled doubleblinded trial surprisingly showed that eplerenone was not superior to placebo for chronic CSCR [29, 30], proving that there must be other missing factors involved in the intricate equation of CSCR pathogenesis. A more recent 
study reported an impaired peripheral endothelial dysfunction in CSCR patients, revealing that it shares the feature of endothelial dysfunction that is common to many cardiovascular diseases [27]. Nevertheless, the precise way this endothelial dysfunction relates to CSCR needs further investigation. Future directions in CSCR etiology should aim at identifying genetic risk factors, high resolution choroidal phenotyping, choroidal, and peripheral endothelial function and cell biology research [31]. Nevertheless, we have to acknowledge some limitations in our study, including a reduced sample of participants, the lack of alcohol and caffeine restraining before their assessment, as well as the lack of control for menstrual cycle and hormonal therapy, although only 8 female patients were evaluated.

\section{Conclusions}

Patients with CSCR presented a significantly impaired neurovascular coupling in their brain, when compared to controls. This finding supports the theory that the pathogenesis of CSCR might supersede a local choroidal vascular disorder and is possibly the reflection of a broader vascular dysfunction. Nevertheless, several questions remain unsolved, rendering the need for further investigation on local and systemic hemodynamics in this pathology.

\section{Statement of Ethics}

Published research complies with the guidelines for human studies and the research was conducted ethically in accordance with the World Medical Association Declaration of Helsinki. Participants gave their written informed consent (as described on the manuscript body) and the study protocol was approved by the Ethics Committee for Health of Centro Hospitalar Universitário de São João, EPE/Faculty of Medicine of University of Porto, Portugal (project approval number: 52/2016).

\section{Conflict of Interest Statement}

Angela Carneiro: participation in advisory boards for Allergan, Alimera, Bayer, Novartis, and Roche. The other authors have no financial disclosures or conflicts of interest to declare.

\section{Funding Sources}

This research did not receive any specific grant from funding agencies in the public, commercial, or not-for-profit sectors for preparation of data or the manuscript.

\section{Author Contributions}

Susana Penas - protocol design and conception; acquisition, analysis, and interpretation of data; and manuscript draft. Pedro Castro - protocol design and conception; acquisition, analysis, and interpretation of data; and manuscript draft. Gilberto Pereira - acquisition of data. Ana Marta Oliveira - protocol design and conception. Angela Carneiro - critical review of the manuscript. Amandio Sousa - critical review of the manuscript. Elsa Azevedo analysis and interpretation of data; critical review of manuscript. Fernando Falcão-Reis - analysis and interpretation of data, and critical review of the manuscript.

\section{Data Availability Statement}

All data generated or analyzed during this study are included in this article. Further enquiries can be directed to the corresponding author.

\section{References}

1 Yannuzzi LA. Type A behavior and central serous chorioretinopathy. Retina. 2012;32(Suppl 1):709.

2 Pang CE, Freund KB. Pachychoroid neovasculopathy. Retina. 2015;35:1-9.

3 Warrow DJ, Hoang QV, Freund KB. Pachychoroid pigment epitheliopathy. Retina. 2013;33:1659-72.

4 Cheung CMG, Lee WK, Koizumi H, Dansingani K, Lai TYY, Freund KB. Pachychoroid disease. Eye. 2019;33(1):14-33.

5 Daruich A, Matet A, Dirani A, Bousquet E, Zhao M, Farman N, et al. Central serous chorioretinopathy: recent findings and new physiopathology hypothesis. Prog Retin Eye Res. 2015;48:82-118.
6 Tsai DC, Chen SJ, Huang CC, Chou P, Chung $\mathrm{CM}$, Chan WL, et al. Risk of central serous chorioretinopathy in adults prescribed oral corticosteroids: a population-based study in Taiwan. Retina. 2014;34:1867-74.

7 Chang YS, Weng SF, Chang C, Wang JJ, Wang JY, Jan RL. Associations between topical ophthalmic corticosteroids and central serous chorioretinopathy: a Taiwanese population-based study. Invest Ophthalmol Vis Sci. 2015;56:4083-9.

8 Bernasconi P, Messmer E, Bernasconi A, Thölen A. Assessment of the sympatho-vagal interaction in central serous chorioretinopathy measured by power spectral analysis of heart rate variability. Graefes Arch Clin Exp Ophthalmol. 1998;236:571-6.
9 Tewari HK, Gadia R, Kumar D, Venkatesh P, Garg SP. Sympathetic-parasympathetic activity and reactivity in central serous chorioretinopathy: a case-control study. Invest Ophthalmol Vis Sci. 2006;47:3474-8.

10 Chen SN, Chen YC, Lian I. Increased risk of coronary heart disease in male patients with central serous chorioretinopathy: results of a population-based cohort study. Br J Ophthalmol. 2014;98:110-4.

11 Tsai DC, Huang CC, Chen SJ, Chou P, Chung $\mathrm{CM}$, Chan WL, et al. Central serous chorioretinopathy and risk of ischaemic stroke: a population-based cohort study. Br J Ophthalmol. 2012;96:1484-8. 
12 Spielberger CD, Gorsuch RL, Lushene R, Vagg PR, Jacobs GA. Manual for the StateTrait Anxiety inventory. Palo Alto, CA: Consulting Psychologists Press; 1983.

13 Santos AS, Silva R. A construção e validação da forma experimental portuguesa do inventário de ansiedade estado-traço-forma Y (Spielberger, 1980): primeiros dados. Rev Port Psicol. 1997;32.

14 Silva DR. Inventário de estado-traço de ansiedade (STAI). In: Gonçalves MRS JM, Almeida LS, Machado C, editors. Avaliação psicológica. Coimbra: Quarteto; 2006. p. 45-60.

15 Madureira J, Castro P, Azevedo E. Demographic and systemic hemodynamic influences in mechanisms of cerebrovascular regulation in healthy adults. J Stroke Cerebrovasc Dis. 2017;26:500-8.

16 Claassen JA, Meel-van den Abeelen AS, Simpson DM, Panerai RB. Transfer function analysis of dynamic cerebral autoregulation: a white paper from the International Cerebral Autoregulation Research Network. J Cereb Blood Flow Metab. 2016;36:665-80.

17 Zhang R, Zuckerman JH, Giller CA, Levine $\mathrm{BD}$. Transfer function analysis of dynamic cerebral autoregulation in humans. Am J Physiol. 1998;274:H233-41.

18 Meel-van den Abeelen AS, van Beek AH, Slump CH, Panerai RB, Claassen JA. Transfer function analysis for the assessment of cerebral autoregulation using spontaneous oscillations in blood pressure and cerebral blood flow. Med Eng Phys. 2014;36:563-75.
19 Sorond FA, Kiely DK, Galica A, Moscufo N, Serrador JM, Iloputaife I, et al. Neurovascular coupling is impaired in slow walkers: the MOBILIZE Boston Study. Ann Neurol. 2011;70: 213-20.

20 Castro P, Azevedo E, Sorond F. Cerebral autoregulation in stroke. Curr Atheroscler Rep. 2018;20:37.

21 Tomasso L, Benatti L, Rabiolo A, Carnevali A, Zucchiatti I, Querques L, et al. Retinal vessels functionality in eyes with central serous chorioretinopathy. $\mathrm{Br}$ J Ophthalmol. 2018;102: 210-4.

22 Iadecola C. The neurovascular unit coming of age: a journey through neurovascular coupling in health and disease. Neuron. 2017;96: 17-42.

23 Drake CT, Iadecola C. The role of neuronal signaling in controlling cerebral blood flow. Brain Lang. 2007;102:141-52.

24 Zhao Z, Ong LK, Johnson S, Nilsson M, Walker FR. Chronic stress induced disruption of the peri-infarct neurovascular unit following experimentally induced photothrombotic stroke. J Cereb Blood Flow Metab. 2017;37: 3709-24.

25 Longden TA, Dabertrand F, Hill-Eubanks DC, Hammack SE, Nelson MT. Stress-induced glucocorticoid signaling remodels neurovascular coupling through impairment of cerebrovascular inwardly rectifying $\mathrm{K}+$ channel function. Proc Natl Acad Sci U S A. 2014; 111:7462-7.
26 Azevedo E, Castro P, Santos R, Freitas J, Coelho $\mathrm{T}$, Rosengarten $\mathrm{B}$, et al. Autonomic dysfunction affects cerebral neurovascular coupling. Clin Auton Res. 2011;21:395-403.

27 Wang NK, Fu Y, Wang JP, Kang EY, Wu AL, Tseng YJ, et al. Peripheral vascular endothelial dysfunction in central serous chorioretinopathy. Invest Ophthalmol Vis Sci. 2017; 58(11):4524-9.

28 Hsu HJ, Lee CY, Chao SC, Nien CW, Tzeng SH, Huang JY, et al. The correlation of central serous chorioretinopathy and subsequent cardiovascular diseases of different types: a population-based cohort study. Int J Environ Res Public Health. 2019;16(24):5099.

29 Willcox A, Culliford L, Ellis L, Rogers CA, Cree A, Chakravarthy U, et al. Clinical efficacy of eplerenone versus placebo for central serous chorioretinopathy: study protocol for the VICI randomised controlled trial. Eye. 2019;33:295-303.

30 Lotery A, Sivaprasad S, O'Connell A, Harris RA, Culliford L, Ellis L, et al. Eplerenone for chronic central serous chorioretinopathy in patients with active, previously untreated disease for more than 4 months (VICI): a randomised, double-blind, placebo-controlled trial. Lancet. 2020;395:294-303.

31 Kaye R, Chandra S, Sheth J, Boon CJF, Sivaprasad S, Lotery A. Central serous chorioretinopathy: an update on risk factors, pathophysiology and imaging modalities. Prog Retin Eye Res. 2020 Nov;79:100865. 